International Journal of Pure and Applied Mathematics

Volume 92 No. 4 2014, 459-469

ISSN: 1311-8080 (printed version); ISSN: 1314-3395 (on-line version)

url: http://www.ijpam.eu

doi: http://dx.doi.org/10.12732/ijpam.v92i4.2

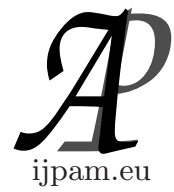

\title{
ON FAINTLY SEMIGENERALIZED $\alpha$-CONTINUOUS FUNCTIONS
}

\author{
N. Rajeah ${ }^{1}$, G. Shanmugam ${ }^{2} \S$ \\ ${ }^{1}$ Department of Mathematics \\ Rajah Serfoji Govt. College \\ Thanjavur, 613005, Tamilnadu, INDIA \\ ${ }^{2}$ Department of Mathematics \\ Jeppiaar Engineering College \\ Chennai, 600119, Tamilnadu, INDIA
}

\begin{abstract}
In this paper we introduce and study a new class of functions called faintly semigeneralized $\alpha$-continuous functions in topological spaces.
\end{abstract}

AMS Subject Classification: 54D10

Key Words: $s g \alpha$-open sets, faintly $s g \alpha$-continuous functions

\section{Introduction}

Recent progress in the study of characterizations and generalizations of continuity, compactness, connectedness, separation axioms etc. has been done by means of several generalized closed sets. The first step of generalizing closed set was done by Levine in 1970 [3]. The notion of generalized closed sets has been studied extensively in recent years by many topologists because generalized closed sets are the only natural generalization of closed sets. More importantly, they also suggest several new properties of topological spaces. As generalization of closed sets, semigeneralized $\alpha$-closed sets were introduced and studied by Rajesh and Krsteska [8]. In this paper, we introduce and study a new class of functions called faintly semigeneralized $\alpha$-continuous functions in topological spaces.

Received: April 11, 2013

(C) 2014 Academic Publications, Ltd. url: www.acadpubl.eu

$\S$ Correspondence author 


\section{Preliminaries}

Throughout the paper $(X, \tau)$ and $(Y, \sigma)$ (or simply $X$ and $Y$ ) represent topological spaces on which no separation axioms are assumed unless otherwise mentioned. For a subset $A$ of a space $(X, \tau), \operatorname{Cl}(A), \operatorname{Int}(A)$ and $A^{c}$ denote the closure of $A$, the interior of $A$ and the complement of $A$ in $X$, respectively. A point $x \in X$ is called a $\theta$-cluster point of $A$ if $\operatorname{Cl}(V) \cap A \neq \emptyset$ for every open set $V$ of $X$ containing $x$. The set of all $\theta$-cluster points of $A$ is called the $\theta$-closure of $A$ and is denoted by $\mathrm{Cl}_{\theta}(A)$. If $A=\mathrm{Cl}_{\theta}(A)$, then $A$ is said to be $\theta$-closed. The complement of $\theta$-closed set is said to be $\theta$-open. The union of all $\theta$-open sets contained in a subset $A$ is called the $\theta$-interior of $A$ and is denoted by $\operatorname{Int}_{\theta}(A)$. It follows from [16] that the collection of $\theta$-open sets in a topological space $(X, \tau)$ forms a topology $\tau_{\theta}$ on $X$.

Definition 1. A subset $A$ of a space $(X, \tau)$ is called :

(i) semiopen [4] if $A \subseteq \mathrm{Cl}(\operatorname{Int}(A))$,

(ii) $\alpha$-open [6] if $A \subseteq \operatorname{Int}(\mathrm{Cl}(\operatorname{Int}(A)))$.

The complement of an $\alpha$-open set is called an $\alpha$-closed set. The $\alpha$-closure of a subset $A$ of $X$, denoted by $\alpha \operatorname{Cl}(A)$ is defined to be the intersection of all $\alpha$-closed sets of $X$ containing $A$.

Definition 2. A subset $A$ of a space $(X, \tau)$ is called semigeneralized $\alpha$ closed (briefly $s g \alpha$-closed) [8] if $\alpha \mathrm{Cl}(A) \subseteq U$ whenever $A \subseteq U$ and $U$ is semiopen in $(X, \tau)$. The complement of a $s g \alpha$-closed set is called a $s g \alpha$-open set. The family of all $s g \alpha$-open (resp. $s g \alpha$-closed) subsets of a space $(X, \tau)$ is denoted by $\operatorname{sg} \alpha O(X)$ (resp. $\operatorname{sg} \alpha C(X))$.

Definition 3. [8] The intersection (union) of all $s g \alpha$-closed ( $s g \alpha$-open) sets containing (contained in) $A$ is called the $s g \alpha$-closure ( $s g \alpha$-interior) of $A$ and is denoted by $s g \alpha-\mathrm{Cl}(A)(s g \alpha-\operatorname{Int}(A))$. A set $A$ is $s g \alpha$-closed if and only if $\operatorname{sg} \alpha-\mathrm{Cl}(A)=A$.

Definition 4. A function $f:(X, \tau) \rightarrow(Y, \sigma)$ is said to be

(i) faintly continuous [5] if $f^{-1}(V)$ is open in $X$ for every $\theta$-open set $V$ of $Y$,

(ii) $s g \alpha$-continuous [10] if for each $x \in X$ and each open set $V$ of $Y$ containing $f(x)$, there exists $U \in \operatorname{sg} \alpha O(X, x)$ such that $f(U) \subset V$,

(iii) weakly $s g \alpha$-continuous [11] if for each $x \in X$ and each open set $V$ of $Y$ containing $f(x)$, there exists $U \in \operatorname{sg\alpha } O(X, x)$ such that $f(U) \subset \mathrm{Cl}(V)$, 
(iv) almost $s g \alpha$-continuous [10] if for each $x \in X$ and each open set $V$ of $Y$ containing $f(x)$, there exists $U \in \operatorname{sg} \alpha O(X, x)$ such that $f(U) \subset \operatorname{Int}(\mathrm{Cl}(V))$,

(v) slightly $s g \alpha$-continuous [12] if for each $x \in X$ and each clopen set $V$ of $Y$ containing $f(x)$, there exists $U \in \operatorname{sg\alpha } O(X, x)$ such that $f(U) \subset V$.

\section{Faintly $s g \alpha$-Continuous Functions}

Definition 5. A function $f:(X, \tau) \rightarrow(Y, \sigma)$ is called faintly $s g \alpha-$ continuous at a point $x \in X$ if for each $\theta$-open set $V$ of $Y$ containing $f(x)$, there exists $U \in \operatorname{sg} \alpha O(X, x)$ such that $f(U) \subset V$. If $f$ has this property at each point of $X$, then it is said to be faintly $s g \alpha$-continuous.

Theorem 6. For a function $f:(X, \tau) \rightarrow(Y, \sigma)$, the following statements are equivalent:

(i) $f$ is faintly sgo-continuous;

(ii) $f^{-1}(V)$ is sgo-open in $X$ for every $\theta$-open set $V$ of $Y$;

(iii) $f^{-1}(F)$ is sgo-closed in $X$ for every $\theta$-closed subset $F$ of $Y$;

(iv) $f:(X, \operatorname{sg} \alpha O(X)) \rightarrow\left(Y, \sigma_{\theta}\right)$ is continuous;

(v) $f:(X, s g \alpha O(X)) \rightarrow(Y, \sigma)$ is faintly continuous;

(vi) $f:(X, \tau) \rightarrow\left(Y, \sigma_{\theta}\right)$ is sgo-continuous.

(vii) $s g \alpha-\mathrm{Cl}\left(f^{-1}(B)\right) \subseteq f^{-1}\left(\mathrm{Cl}_{\theta}(B)\right)$ for every subset $B$ of $Y$;

(viii) $f^{-1}\left(\operatorname{Int}_{\theta}(G)\right) \subseteq s g \alpha-\operatorname{Int}\left(f^{-1}(G)\right)$ for every subset $G$ of $Y$.

Proof. (i) $\Rightarrow$ (ii): Let $V$ be an $\theta$-open set of $Y$ and $x \in f^{-1}(V)$. Since $f(x) \in V$ and $f$ is faintly sgo-continuous, there exists $U \in \operatorname{sg} \alpha O(X, x)$ such that $f(U) \subset V$. It follows that $x \in U \subset f^{-1}(V)$. Hence $f^{-1}(V)$ is sgo-open in $X$.

(ii) $\Rightarrow($ i): Let $x \in X$ and $V$ be an $\theta$-open set of $Y$ containing $f(x)$. By (ii), $f^{-1}(V)$ is a $s g \alpha$-open set containing $x$. Take $U=f^{-1}(V)$. Then $f(U) \subset V$. This shows that $f$ is faintly $s g \alpha$-continuous.

(ii) $\Rightarrow$ (iii): Let $V$ be any $\theta$-closed set of $Y$. Since $Y \backslash V$ is an $\theta$-open set, by (ii), it follows that $f^{-1}(Y \backslash V)=X \backslash f^{-1}(V)$ is sgo-open. This shows that $f^{-1}(V)$ is $s g \alpha$-closed in $X$. 
(iii) $\Rightarrow$ (ii): Let $V$ be an $\theta$-open set of $Y$. Then $Y \backslash V$ is $\theta$-closed in $Y$. By (iii), $f^{-1}(Y \backslash V)=X \backslash f^{-1}(V)$ is $s g \alpha$-closed and thus $f^{-1}(V)$ is $s g \alpha$-open.

(i) $\Leftrightarrow($ iv $) \Leftrightarrow(\mathrm{v}) \Leftrightarrow($ vi) and (iii) $\Leftrightarrow($ vii $) \Leftrightarrow($ viii) are Obvious.

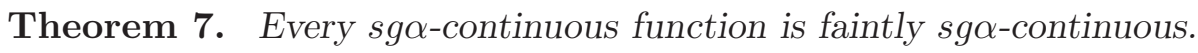

Proof. The proof is clear.

The following example shows that the converse of Theorem 7 is not true in general.

Example 8. Let $X=\{a, b, c\}, \tau=\{\emptyset,\{a\}, X\}$ and $\sigma=\{\emptyset,\{b\}, X\}$. Then the identity function $f:(X, \tau) \rightarrow(X, \sigma)$ is faintly $s g \alpha$-continuous but not $s g \alpha$-continuous.

Theorem 9. If a function $f:(X, \tau) \rightarrow(Y, \sigma)$ is weakly sgo-continuous, then it is faintly sga-continuous.

Proof. Let $x \in X$ and $V$ be a $\theta$-open set containing $f(x)$. Then, there exists an open set $W$ such that $f(x) \in W \subset \mathrm{Cl}(W) \subset V$. Since $f$ is weakly $s g \alpha$-continuous, there exists a $s g \alpha$-open set $U$ containing $x$ such that $f(U) \subset$ $\mathrm{Cl}(W) \subset V$. Therefore, $f$ is faintly $s g \alpha$-continuous.

The converse of Theorem 9 is not true in general as shown in the following example.

Example 10. Let $X=\{a, b, c\}, \tau=\{\emptyset,\{a, b\}, X\}$ and $\sigma=\{\emptyset,\{b\}$, $\{c\},\{b, c\}, X\}$. Clearly the identity function $f:(X, \tau) \rightarrow(X, \sigma)$ is faintly $s g \alpha$-continuous but not weakly $s g \alpha$-continuous.

Definition 11. A topological space $(X, \tau)$ is said to be almost-regular [14] if for each regular closed set $F$ of $X$ and each point $x \notin F$, there exist disjoint open sets $U$ and $V$ of $X$ such that $x \notin U$ and $F \subset V$.

Theorem 12. [10] For a function $f:(X, \tau) \rightarrow(Y, \sigma)$, the following are equivalent:

(i) $f$ is almost sg $\alpha$-continuous at $x \in X$;

(ii) for every regular open set $V$ containing $f(x)$, there exists $U \in \operatorname{sg\alpha } O(X, x)$ such that $f(U) \subset V$.

Theorem 13. If a function $f:(X, \tau) \rightarrow(Y, \sigma)$ is faintly sgo-continuous and $(Y, \sigma)$ is almost-regular, then $f$ is almost sgo-continuous. 
Proof. Let $x \in X$ and $V$ be any regular open set of $(Y, \sigma)$ containing $f(x)$. Since every regular open set in an almost-regular space is $\theta$-open [5], $V$ is $\theta$ open. Since $f$ is faintly $s g \alpha$-continuous, there exists $U \in \operatorname{sg} \alpha O(X, x)$ such that $f(U) \subset V$. It follows from Theorem 6 that $f$ is almost $s g \alpha$-continuous.

Corollary 14. Let $(Y, \sigma)$ be an almost-regular space. Then, for a function $f:(X, \tau) \rightarrow(Y, \sigma)$, the following properties are equivalent:

(i) $f$ is almost sgo-continuous;

(ii) $f$ is weakly sgo-continuous;

(iii) $f$ is faintly sgo-continuous.

Proof. The proof follows from Theorems 9 and 13.

Theorem 15. If a function $f:(X, \tau) \rightarrow(Y, \sigma)$ is faintly sgo-continuous and $(Y, \sigma)$ is a regular space, then $f$ is sgo-continuous.

Proof. Let $V$ be any open set of $Y$. Since $Y$ is regular, $V$ is $\theta$-open in $Y$. Since $f$ is faintly $s g \alpha$-continuous, by Theorem 6 , we have $f^{-1}(V)$ is $s g \alpha$-open and hence $f$ is $s g \alpha$-continuous.

Theorem 16. If a function $f:(X, \tau) \rightarrow(Y, \sigma)$ is faintly sgo-continuous, then it is slightly sgo-continuous.

Proof. Let $x \in X$ and $V$ be any clopen subset of $(Y, \sigma)$ containing $f(x)$. Then $V$ is $\theta$-open in $Y$. Since $f$ is faintly sgo-continuous, there exists $U \in$ $\operatorname{sg} \alpha O(X, x)$ containing $x$ such that $f(U) \subset V$. This shows that $f$ is slightly $\operatorname{sg} \alpha$-continuous.

Definition 17. Let $(X, \tau)$ be a topological space. Since the intersection of two clopen sets of $(X, \tau)$ is clopen, the clopen sets of $(X, \tau)$ may be use as a base for a topology for $X$. This topology is called the ultra-regularization of $\tau$ [7] and is denoted by $\tau_{u}$. A topological space $(X, \tau)$ is said to be ultra-regular [1] if $\tau=\tau_{u}$.

Theorem 18. Let $(Y, \sigma)$ be an ultra-regular space. Then, for a function $f:(X, \tau) \rightarrow(Y, \sigma)$, the following properties are equivalent:

(i) $f$ is sgo-continuous;

(ii) $f$ is almost sg $\alpha$-continuous;

(iii) $f$ is weakly sgo-continuous; 
(iv) $f$ is faintly sgo-continuous;

(v) $f$ is slightly sgo-continuous.

Proof. The proof follows from definitions and Theorems 9, 13 and Theorem 16.

Definition 19. A $s g \alpha$-frontier of a subset $A$ of $(X, \tau)$ is $\operatorname{sg} \alpha-\operatorname{Fr}(A)=$ $s g \alpha-\mathrm{Cl}(A) \cap s g \alpha-\mathrm{Cl}(X \backslash A)$.

Theorem 20. The set of all points $x \in X$ in which a function $f:(X, \tau) \rightarrow$

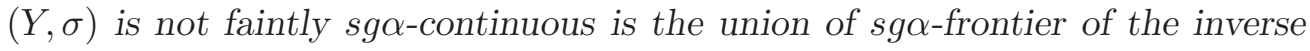
images of $\theta$-open sets containing $f(x)$.

Proof. Suppose that $f$ is not faintly $s g \alpha$-continuous at $x \in X$. Then there exists an $\theta$-open set $V$ of $Y$ containing $f(x)$ such that $f(U)$ is not contained in $V$ for each $U \in \operatorname{sg\alpha } O(X)$ containing $x$ and hence $x \in \theta-\mathrm{Cl}\left(X \backslash f^{-1}(V)\right)$. On the otherhand, $x \in f^{-1}(V) \subset \operatorname{sg} \alpha-\mathrm{Cl}\left(f^{-1}(V)\right)$ and hence $x \in \operatorname{sg\alpha }-F r\left(f^{-1}(U)\right)$. Conversely, suppose that $f$ is faintly $s g \alpha$-continuous at $x \in X$ and let $V$ be a $\theta$-open set of $Y$ containing $f(x)$. Then there exists $U \in \operatorname{sg\alpha } O(X)$ containing $x$ such that $U \subset f^{-1}(V)$. Hence $x \in \operatorname{Int}_{\theta}\left(f^{-1}(V)\right)$. Therefore, $x \in s g \alpha-$ $\operatorname{Fr}\left(f^{-1}(V)\right)$ for each open set $V$ of $Y$ containing $f(x)$.

Theorem 21. Let $f:(X, \tau) \rightarrow(Y, \sigma)$ be a function and $g:(X, \tau) \rightarrow$ $(X \times Y, \tau \times \sigma)$ the graph function of $f$, defined by $g(x)=(x, f(x))$ for every

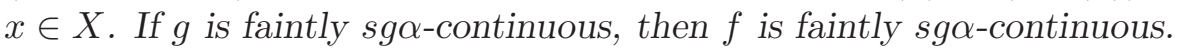

Proof. Let $U$ be an $\theta$-open set in $(Y, \sigma)$, then $X \times U$ is a $\theta$-open set in $X \times Y$. It follows that $f^{-1}(U)=g^{-1}(X \times U) \in s g \alpha O(X)$. This shows that $f$ is faintly $s g \alpha$-continuous.

Definition 22. A space $(X, \tau)$ is said to be sgo-connected [9] if $X$ cannot be written as a disjoint union of two nonempty $s g \alpha$-open sets.

Theorem 23. If $f:(X, \tau) \rightarrow(Y, \sigma)$ is a faintly sgo-continuous function and $(X, \tau)$ is a sgo-connected space, then $Y$ is a connected space.

Proof. Assume that $(Y, \sigma)$ is not connected. Then there exist nonempty open sets $V_{1}$ and $V_{2}$ such that $V_{1} \cap V_{2}=\emptyset$ and $V_{1} \cup V_{2}=Y$. Hence we have $f^{-1}\left(V_{1}\right) \cap f^{-1}\left(V_{2}\right)=\emptyset$ and $f^{-1}\left(V_{1}\right) \cup f^{-1}\left(V_{2}\right)=X$. Since $f$ is surjective, $f^{-1}\left(V_{1}\right)$ and $f^{-1}\left(V_{2}\right)$ are nonempty subsets of $X$. Since $V_{i}$ is open and closed, $V_{i}$ is $\theta$-open for each $i=1,2$. Since $f$ is faintly $s g \alpha$-continuous, $f^{-1}\left(V_{i}\right) \in$ $\operatorname{sg} \alpha O(X)$. Therefore, $(X, \tau)$ is not $s g \alpha$-connected. This is a contradiction and hence $(Y, \sigma)$ is connected. 
Definition 24. A space $(X, \tau)$ is said to be $s g \alpha$-compact [9] (resp. $\theta$ compact [2]) if each $s g \alpha$-open (resp. $\theta$-open) cover of $X$ has a finite subcover.

Theorem 25. The surjective faintly sgo-continuous image of a $s g \alpha$ compact space is $\theta$-compact.

Proof. Let $f:(X, \tau) \rightarrow(Y, \sigma)$ be a faintly $s g \alpha$-continuous function from a $s g \alpha$-compact space $X$ onto a space $Y$. Let $\left\{G_{\alpha}: \alpha \in I\right\}$ be any $\theta$-open cover of $Y$. Since $f$ is faintly $s g \alpha$-continuous, $\left\{f^{-1}\left(G_{\alpha}\right): \alpha \in I\right\}$ is a $s g \alpha$-open cover of $X$. Since $X$ is $s g \alpha$-compact, there exists a finit subcover $\left\{f^{-1}\left(G_{i}\right): i=1\right.$, $2, \ldots . n\}$ of $X$. Then it follows that $\left\{G_{i}: i=1,2, \ldots n\right\}$ is a finite subfamily which cover $Y$. Hence $Y$ is $\theta$-compact.

\section{Separation Axioms}

Definition 26. A topological space $(X, \tau)$ is said to be:

(i) $s g \alpha-T_{1}$ [13] (resp. $\theta-T_{1}$ ) if for each pair of distinct points $x$ and $y$ of $X$, there exists $s g \alpha$-open (resp. $\theta$-open) sets $U$ and $V$ containing $x$ and $y$, respectively such that $y \notin U$ and $x \notin V$.

(ii) $s g \alpha-T_{2}[13]$ (resp. $\theta-T_{2}$ [15]) if for each pair of distinct points $x$ and $y$ in $X$, there exists disjoint $s g \alpha$-open (resp. $\theta$-open) sets $U$ and $V$ in $X$ such that $x \in U$ and $y \in V$.

Theorem 27. If $f:(X, \tau) \rightarrow(Y, \sigma)$ is faintly sgo-continuous injection and $Y$ is a $\theta-T_{1}$ space, then $X$ is a $s g \alpha-T_{1}$ space.

Proof. Suppose that $Y$ is $\theta-T_{1}$. For any distinct points $x$ and $y$ in $X$, there exist $V, W \in \sigma_{\theta}$ such that $f(x) \in V, f(y) \notin V, f(x) \notin W$ and $f(y) \in W$. Since $f$ is faintly $s g \alpha$-continuous, $f^{-1}(V)$ and $f^{-1}(W)$ are $s g \alpha$-open subsets of $(X, \tau)$ such that $x \in f^{-1}(V), y \notin f^{-1}(V), x \notin f^{-1}(W)$ and $y \in f^{-1}(W)$. This shows that $X$ is $s g \alpha-T_{1}$.

Theorem 28. If $f:(X, \tau) \rightarrow(Y, \sigma)$ is faintly sgo-continuous injection and $Y$ is a $\theta-T_{2}$ space, then $X$ is a $s g \alpha-T_{2}$ space.

Proof. Suppose that $Y$ is $\theta-T_{2}$. For any pair of distinct points $x$ and $y$ in $X$, there exist disjoint $\theta$-open sets $U$ and $V$ in $Y$ such that $f(x) \in U$ and $f(y) \in V$. Since $f$ is faintly $s g \alpha$-continuous, $f^{-1}(U)$ and $f^{-1}(V)$ are $s g \alpha$-open in $X$ containing $x$ and $y$, respectively. Therefore, $f^{-1}(U) \cap f^{-1}(V)=\emptyset$ because $U \cap V=\emptyset$. This shows that $X$ is $s g \alpha-T_{2}$. 
Recall that for a function $f:(X, \tau) \rightarrow(Y, \sigma)$, the subset $\{(x, f(x)): x \in X\}$ $\subset X \times Y$ is called the graph of $f$ and is denoted by $G(f)$.

Definition 29. A graph $G(f)$ of a function $f:(X, \tau) \rightarrow(Y, \sigma)$ is said to be $\theta$-sg $\alpha$-closed if for each $(x, y) \in(X \times Y) \backslash G(f)$, there exist $U \in \operatorname{sg\alpha } O(X, x)$ and $V \in \sigma_{\theta}$ containing $y$ such that $(U \times V) \cap G(f)=\emptyset$.

Lemma 30. A graph $G(f)$ of a function $f:(X, \tau) \rightarrow(Y, \sigma)$ is $\theta$-sg $\alpha$ closed in $X \times Y$ if and only if for each $(x, y) \in(X \times Y) \backslash G(f)$, there exist $U$ $\in \operatorname{sg\alpha } O(X, x)$ and $V \in \sigma_{\theta}$ containing $y$ such that $f(U) \cap V=\emptyset$.

Proof. It is an immediate consequence of Definition 29.

Theorem 31. If $f:(X, \tau) \rightarrow(Y, \sigma)$ is faintly sgo-continuous function and $(Y, \sigma)$ is $\theta-T_{2}$, then $G(f)$ is $\theta$-sg $\alpha$-closed.

Proof. Let $(x, y) \in(X \times Y) \backslash G(f)$, then $f(x) \neq y$. Since $Y$ is $\theta-T_{2}$, there exist $\theta$-open sets $V$ and $W$ in $Y$ such that $f(x) \in V, y \in W$ and $V \cap W=\emptyset$. Since $f$ is faintly sgo-continuous, $f^{-1}(V) \in \operatorname{sg} \alpha O(X, x)$. Take $U=f^{-1}(V)$. We have $f(U) \subset V$. Therefore, we obtain $f(U) \cap V=\emptyset$. This shows that $G(f)$ is $\theta$-sg $\alpha$-closed.

Theorem 32. Let $f:(X, \tau) \rightarrow(Y, \sigma)$ has $\theta$-sg $\alpha$-closed graph $G(f)$. If $f$ is a faintly sgo-continuous injection, then $(X, \tau)$ is $s g \alpha-T_{2}$.

Proof. Let $x$ and $y$ be any two distinct points of $X$. Then since $f$ is injective, we have $f(x) \neq f(y)$. Then, we have $(x, f(y)) \in(X \times Y) \backslash G(f)$. By Lemma $30, U \in \operatorname{sg\alpha } O(X)$ and $V \in \sigma_{\theta}$ such that $(x, f(y)) \in U \times V$ and $f(U) \cap V=$ $\emptyset$. Hence $U \cap f^{-1}(V)=\emptyset$ and $y \notin U$. Since $f$ is faintly $s g \alpha$-continuous, there exists $W \in \operatorname{sg\alpha } O(X, y)$ such that $f(W) \subset V$. Therefore, we have $f(U) \cap f(W)$ $=\emptyset$. Since $f$ is injective, we obtain $U \cap W=\emptyset$. This implies that $(X, \tau)$ is $\operatorname{sg} \alpha-T_{2}$.

Definition 33. A function $f:(X, \tau) \rightarrow(Y, \sigma)$ is said to have a sgacclosed graph if for each $(x, y) \in(X \times Y) \backslash G(f)$, there exists $U \in \operatorname{sg\alpha } O(X, x)$ and an open set $V$ of $Y$ containing $y$ such that $(U \times \mathrm{Cl}(V)) \cap G(f)=\emptyset$.

Lemma 34. Let $f:(X, \tau) \rightarrow(Y, \sigma)$ be a function. Then its graph $G(f)$ is sgac-closed in $X \times Y$ if and only if for each point $(x, y) \in(X \times Y) \backslash G(f)$, there exist $U \in \operatorname{sg\alpha } O(X)$ and $V \in \sigma$ containing $x$ and $y$, respectively, such that $f(U) \cap \mathrm{Cl}(V)=\emptyset$.

Proof. It is an immediate consequence of Definition 33. 
Theorem 35. If $f:(X, \tau) \rightarrow(Y, \sigma)$ is a surjective function with a sgacclosed graph, then $(Y, \sigma)$ is Hausdorff.

Proof. Let $y_{1}$ and $y_{2}$ be any distinct points of $Y$. Then since $f$ is surjective, there exists $x_{1} \in X$ such that $f\left(x_{1}\right)=y_{1}$; hence $\left(x_{1}, y_{2}\right) \in(X \times Y) \backslash G(f)$. Since $G(f)$ is $s g \alpha c$-closed, there exist $U \in \operatorname{sg\alpha } O\left(X, x_{1}\right)$ and an open set $V$ of $Y$ containing $y_{2}$ such that $f(U) \cap \mathrm{Cl}(V)=\emptyset$. Therefore, we have $y_{1}=f\left(x_{1}\right)$ $\in f(U) \subset Y \backslash \mathrm{Cl}(V)$. Then there exists an open set $H$ of $Y$ such that $y_{1} \in H$ and $H \cap V=\emptyset$. Moreoever, we have $y_{2} \in V$ and $V$ is open in $Y$. This shows that $Y$ is Hausdorff.

Theorem 36. If $f:(X, \tau) \rightarrow(Y, \sigma)$ has an $\theta$-sgo-closed graph, it has a sgac-closed graph.

Proof. Let $x \in X$ and $y \neq f(x)$, then $(x, y) \in(X \times Y) \backslash G(f)$. By Lemma 30 , there exist $U \in \operatorname{sg\alpha } O(X, x)$ and a $\theta$-open set $V$ containing $y$ such that $f(U) \cap V=\emptyset$. Since $V$ is $\theta$-open, there exists an open set $V_{0}$ such that $y \in V_{0}$ $\subset \mathrm{Cl}\left(V_{0}\right) \subset V$ so that $f(U) \cap \mathrm{Cl}\left(V_{0}\right)=\emptyset$. It follows from Lemma 34 that the graph of $f$ is $s g \alpha c$-closed.

Corollary 37. If $f:(X, \tau) \rightarrow(Y, \sigma)$ is a faintly sgo-continuous and $(Y, \sigma)$ is $\theta-T_{2}$, then $f$ has a sgac-closed graph.

Proof. The proof follows from Theorems 31 and 36.

Theorem 38. If $f:(X, \tau) \rightarrow(Y, \sigma)$ has the $\theta$-sgo-closed graph, then $f(K)$ is $\theta$-closed in $(Y, \sigma)$ for each subset $K$ which is sgo-compact relative to $X$.

Proof. Suppose that $y \notin f(K)$. Then $(x, y) \notin G(f)$ for each $x \in K$. Since $G(f)$ is $\theta$-sg $\alpha$-closed, there exist $U_{x} \in \operatorname{sg\alpha } O(X, x)$ and a $\theta$-open set $V_{x}$ of $Y$ containing $y$ such that $f\left(U_{x}\right) \cap V_{x}=\emptyset$ by Lemma 30. The family $\left\{U_{x}: x \in K\right\}$ is a cover of $K$ by $s g \alpha$-open sets. Since $K$ is $s g \alpha$-compact relative to $(X, \tau)$, there exists a finite subset $K_{0}$ of $K$ such that $K \subset \cup\left\{U_{x}: x \in K_{0}\right\}$. Set $V=$ $\cap\left\{V_{x}: x \in K_{0}\right\}$. Then $V$ is a $\theta$-open set in $Y$ containing $y$. Therefore, we have $f(K) \cap V \subset\left[\cup_{x \in K_{0}} f\left(U_{x}\right)\right] \cap V \subset \cup_{x \in K_{0}}\left[f\left(U_{x}\right) \cap V\right]=\emptyset$. It follows that $y \notin$ $\mathrm{Cl}_{\theta}(f(K))$. Therefore, $f(K)$ is $\theta$-closed in $(Y, \sigma)$.

Corollary 39. If $f:(X, \tau) \rightarrow(Y, \sigma)$ is faintly sgo-continuous and $(Y, \sigma)$ is $\theta$ - $T_{2}$, then $f(K)$ is $\theta$-closed in $(Y, \sigma)$ for each subset $K$ which is sgo-compact relative to $(X, \tau)$.

Proof. The proof follows from Theorems 31 and 38. 


\section{References}

[1] R. L. Ellis, A non-archimedean analogue of the Tietz Urysohn extension theorem, Nederl. Akad. Wetensch. Proc. Ser. A., 70(1967), 332-333.

[2] S. Jafari, Some properties of quasi $\theta$-continuous functions, Far East J. Math. Soc., 6(1998), 689-696.

[3] N. Levine, Generalized closed sets in topology, Rend. Circ. Mat. Palermo, 19(1970), 89-96.

[4] N. Levine, Semi-open sets and semi-continuity in topological spaces, Amer. Math. Monthly, 70(1963), 36-41.

[5] P. E. Long and L. L. Herrington, The $T_{\theta}$-topology and faintly continuous functions, Kyungpook Math. J., 22(1982), 7-14.

[6] O. Njstad, On some classes of nearly open sets, Pacific J. Math. 15(1965), 961-970.

[7] T. Noiri, On slightly $\beta$-continuous functions, Int. J. Math. Math. Sci, 28(2001), 469-478.

[8] N. Rajesh and K. Biljana, Semigeneralized $\alpha$-closed sets, Antarctica J. Math., 6(1) 2009, 1-12.

[9] N. Rajesh, G. Shanmugam and K. Biljana, Semigeneralized $\alpha$-Continuous Functions (submitted).

[10] N. Rajesh and G. Shanmugam, On Almost semigeneralized $\alpha$-continuous functions (submitted).

[11] N. Rajesh and G. Shanmugam, On Weakly semigeneralized $\alpha$-continuous functions (submitted).

[12] N. Rajesh and G. Shanmugam, On slightly semigeneralized $\alpha$-continuous functions (submitted).

[13] N. Rajesh, G. Shanmugam and K. Biljana, Characterization of $s g \alpha-T_{0}$, $s g \alpha-T_{1}$ and $s g \alpha-T_{2}$ topological spaces (submitted).

[14] A. R. Singal and S. P. Arya, On almost-regular spaces, Glasnik Mat Ser III, 4(24)(1969), 89-99. 
[15] S. Sinharoy and S. Bandyopadhyay, On $\theta$-completely regular and locally (-H-closed spaces, Bull. Cal. Math. Soc., 87(1995), 19-28.

[16] N. V. Velicko, H-closed topological spaces, Amer. Math. Soc. Transl., 78(1968), 103-118. 
